\title{
Phénomène de gigantisme dans une population de Nématodes parasite de Tortue malgache
}

\author{
par Annie J. PETTER et Edouard R. BRYGOo \\ Laboratoire de Zoologie (Vers), associé au C.N.R.S. ( $\mathrm{P}^{\mathrm{r}}$ A.-G. Chabaud), \\ Muséum national d'Histoire naturelle, 57, rue Cuvier, F 75005 Paris, \\ et Institut Pasteur de Madagascar, B.P. 1274, Tananarive, République Malgache
}

\section{Résumé}

Etude de la population de Nématodes du côlon d'une Testudo yniphora Vaillant, espèce localisée dans le Nord-Ouest de Madagascar et complètement isolée des autres Tortues malgaches. Elle est composée d'espèces connues chez les Testudo radiata, mais cinq espèces seulement sont présentes, et on observe par rapport à ces Tortues une augmentation de la taille de l'ensemble de la population.

\section{Summary}

The population of Nematodes from the colon of a Testudo yniphora Vaillant is studied; Testudo yniphora lives in the North-West of Madagascar and is completely isolated from other madagascan tortoises. The population is composed of species known from Testudo radiata, but only five species are present and compared with these tortoises an increase of the size of the species is observed.

Nous avons examiné la population de Nématodes du côlon d'une Testudo yniphora Vaillant (178 BA), ramassée par M. Bourgat, dans la région de Soalala (nord-ouest de Madagascar).

Les Testudo yniphora sont des Tortues localisées dans une petite région du nord-ouest de Madagascar, où elles se trouvent complètement isolées des Testudo radiata Shaw et Pyxis arachnoïdes Bell qui habitent le sud de l'île; il nous a donc paru intéressant de voir si leur population de Nématodes présentait des différences avec celle des autres Tortues malgaches. 
La Tortue examinée possédait peu de parasites (environ 500 individus); nous avons constaté que la population était constituée d'espèces déjà connues chez les Testudo radiata, mais que cinq seulement des espèces de ces Tortues étaient représentées, alors que chez les autres Tortues malgaches, le nombre d'espèces différentes trouvé chez une même Tortue est généralement d'une dizaine (voir Petter, 1966) ; parmi ces cinq espèces, deux seulement sont en abondance: Thelandros pyxis dolichurus et Ortleppnema radiatum; à côté de celles-ci, nous rencontrons quelques femelles de Mehdiella stylosa dollfusi, un mâle de Tachygonetria macrolaimus dessetae, et quelques femelles juvéniles d'Atractis chabaudi.
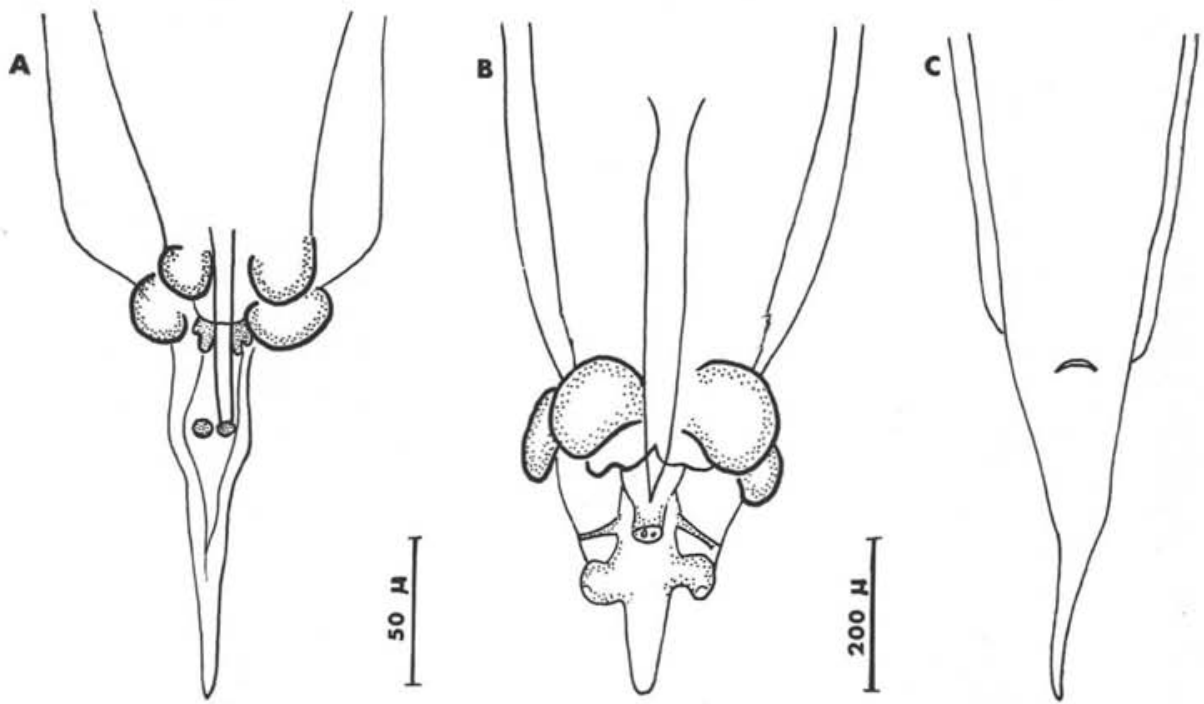

Fig. 1. - Spécimens trouvés chez Testudo yniphora

A. - Extrémité caudale d'un mâle de Thelandros pyxis dolichurus, vue ventrale.

B. - Extrémité caudale d'un mâle de Ortleppnema radiatum, vue ventrale.

C. - Extrémité caudale d'nue femelle de Ortleppnema radiatum, vue ventrale.

(A, B : échelle $50 \mu, C$ : échelle $200 \mu$ )

Au point de vue morphologique, les deux espèces abondantes présentent quelques particularités qui les diffèrencient des spécimens types décrits chez Testudo radiata; ainsi, les mâles de Thelandros pyxis dolichurus ont une pointe caudale moins effilée, et la deuxième paire de papilles cloacales a un aspect plus court et plus robuste (fig. 1, A) ; chez Ortleppnema radiatum, les ailes latérales se prolongent jusqu'au niveau de l'anus chez les femelles (fig. 1, C), et chez les mâles, la pointe caudale est plus courte et plus massive (fig. 1, B).

Cependant, le fait le plus remarquable que l'on observe est la grande taille présentée par l'ensemble de la population; nous donnons, pour mettre le phénomène en 
évidence, les longueurs pour chaque espèce de plusieurs individus pris au hasard, d'une part chez la Testudo yniphora, d'autre part chez une Testudo radiata (101 Q) ; nous n'avons pas mesuré l'espèce Atractis chabaudi puisqu'elle n'est représentée que par quelques femelles juvéniles.

\begin{tabular}{|c|c|c|c|}
\hline & \multicolumn{2}{|c|}{$\begin{array}{c}\text { Spécimens de Testudo yniphora } \\
\text { longueurs en } \mathrm{mm}\end{array}$} & $\begin{array}{c}\text { Spécimens de Testudo radiata } \\
\text { ou Pyxis arachnoödes } \\
\text { longueurs en mm }\end{array}$ \\
\hline \multirow{2}{*}{ Thel. p.d. } & $q$ & $\begin{array}{l}7: 7,1 ; 7,1 ; 7,1 ; 7,1 ; 7,2 ; \\
7,2 ; 7,3 ; 7,4 ; 7,5\end{array}$ & $\begin{array}{l}4,2 ; 4,3 ; 4,3 ; 4,4 ; 4,5 ; 4,6 ; \\
4,6 ; 4,6 ; 4,7 ; 5,5\end{array}$ \\
\hline & $\delta$ & $\begin{array}{l}3,9 ; 4 ; 4 ; 4 ; 4 ; 4,2 ; 4,2 ; \\
4,2 ; 4,2 ; 4,2\end{array}$ & $\begin{array}{l}2,3 ; 2,3 ; 2,4 ; 2,4 ; 2,5 ; 2,6 \text {; } \\
2,6 ; 2,6 ; 2,7 ; 2,9\end{array}$ \\
\hline \multirow{2}{*}{ Ort. $r$. } & $q$ & $\begin{array}{l}6,7 ; 6,9 ; 6,9 ; 7,6 ; 7,3 ; 7,4 ; \\
7,5 ; 7,7 ; 7,7 ; 7,9\end{array}$ & $\begin{array}{l}4,2 ; 4,3 ; 5,2 ; 5,2 ; 5,5 ; 5,6 \text {; } \\
5,7 ; 5,7 ; 5,8 ; 7,1\end{array}$ \\
\hline & $\sigma^{2}$ & 5,$3 ; 5,6 ; 5,9 ; 5,9$ & 3,$8 ; 4,2 ; 4,3 ; 4,7$ \\
\hline Meh. s.d. & q & 6,$3 ; 6,4 ; 6,6 ; 6,7 ; 6,7$ & $\begin{array}{l}4 ; 4,1 ; 4,2 ; 4,3 ; 4,3 ; 4,5 ; \\
4,5 ; 4,6 ; 4,9 ; 4,9\end{array}$ \\
\hline T. m. d. & $\sigma^{2}$ & 3,5 & 2,$1 ; 2,3 ; 2,6 ; 2,6 ; 2,7$ \\
\hline
\end{tabular}

Autant que l'on puisse en juger par l'examen d'une seule Tortue, l'isolement de l'espèce-hôte a donc entraîné deux phénomènes : d'une part, un appauvrissement de la faune, phénomène qui n'est peut-être en partie qu'une apparence due au fait que la Tortue étant peu parasitée, les espèces rares ne se rencontrent pas, d'autre part, une augmentation de la taille des espèces.

Cette constatation est intéressante, car ce double phénomène d'appauvrissement de la faune et d'augmentation de la taille des espèces a déjà été rencontré chez les Testudo pardalis du Swaziland, région d'Afrique du Sud isolée par des montagnes, et dont les Tortues possèdent une faune qui est une image appauvrie de celle des Tortues paléarctiques, l'ensemble de la population étant légèrement plus grand que celui de ces Tortues.

En ce qui concerne les Testudo yniphora, l'accroissement de la taille des espèces étant un caractère d'évolution, nous pouvons conclure que la faune de ces Tortues dérive de celle des Testudo radiata; de plus, étant donné la lenteur et le peu d'amplitude des processus d'évolution chez les Oxyures de Tortues, le fait que la faune se soit modifiée, même légèrement, par rapport à celle des Testudo radiata nous fait supposer que l'isolement de ces Tortues est déjà assez ancien.

\section{Bibliographie}

Petter (A.-J.), 1966. - Equilibre des espèces dans les populations de Nématodes parasites du côlon des Tortues terrestres. Mém. Mus. nat. Hist. Nat., Nlle Série, Série A, Zoologie, 39, 1-252. 